\title{
Effect of integrated nutrient management on the nutrient accumulation and status of post-harvest soil of brinjal (Solanum melongena L.) under Nadia conditions (West Bengal), India
}

\author{
Umalaxmi Thingujam ${ }^{1 *}$, Sajal Pati ${ }^{1}$, Rubina Khanam ${ }^{1}$, Arnab Pari ${ }^{1}$, Krishnendu Ray ${ }^{2}$, \\ Amit Phonglosa ${ }^{3}$ and Kallol Bhattacharyya ${ }^{1}$ \\ ${ }^{1}$ Department of Agricultural Chemistry and Soil Science, Bidhan Chandra Krishi Viswavidyalaya, Mohanpur, Nadia- \\ 741252 (West Bengal), INDIA \\ ${ }^{2}$ Department of Agronomy, Bidhan Chandra Krishi Viswavidyalaya. Mohanpur, Nadia-741252 (West Bengal), INDIA \\ ${ }^{3}$ Department of Soil Science and Agriculture Chemistry, Orissa University of Agriculture \&Technology, Bhubaneswar- \\ 751003 (Orissa), INDIA \\ *Corresponding author. E-mail: thingujam9@gmail.com
}

Received: June 25, 2015; Revised received: December 25, 2015; Accepted: March 1, 2016

\begin{abstract}
A field experiment was carried out at the Central Research Farm, Bidhan Chandra Krishi Viswavidyalaya, West Bengal, India to study the effects of integrated nutrient management on the nutrient accumulation (dry weight recoveries) in brinjal and plant nutrient status of the post- harvest soil of brinjal under Nadia conditions. The results revealed that the treatment consisting of 75\% RDF (RDF i.e. N:P:K:: 125:100:50) + Azospirillum + phosphate solubilising bacteria (PSB) + Borax @ $10 \mathrm{~kg} \mathrm{ha}^{-1}$ recorded the highest oxidizable organic carbon $\left(8.049 \mathrm{~g} \mathrm{~kg}^{-1}\right)$, total nitrogen $\left(1.05 \mathrm{~g} \mathrm{~kg}^{-1}\right)$, available nitrogen $\left(212.67 \mathrm{~g} \mathrm{~kg}^{-1}\right)$, available phosphorus $\left(76.20 \mathrm{~g} \mathrm{~kg}^{-1}\right)$ and available potassium $\left(177.59 \mathrm{~g} \mathrm{~kg}^{-1}\right)$ in the post harvest soils of brinjal. On the other hand, $75 \%$ RDF + Azospirillum + PSB + FeSO $@ 50$ $\mathrm{kg} \mathrm{ha}^{-1}$ recorded the highest available iron $\left(26.14 \mathrm{~kg} \mathrm{ha}^{-1}\right)$ and the treatment consisting of $75 \% \mathrm{RDF}+$ Azospirillum + $\mathrm{PSB}+\mathrm{ZnSO}_{4} @ 25 \mathrm{~kg} \mathrm{ha}^{-1}$ recorded the highest soil available zinc $\left(7.62 \mathrm{~kg} \mathrm{ha}^{-1}\right)$ while $75 \%$ RDF + Azo + PSB + Borax @ $10 \mathrm{~kg} \mathrm{ha}^{-1}$ recorded the highest available Boron content $\left(0.78 \mathrm{~kg} \mathrm{ha}^{-1}\right)$ of the post harvest soil of Brinjal. Highest brinjal yield $\left(14.96 \mathrm{t} \mathrm{ha}^{-1}\right)$ was supported by the treatment consisting of $75 \%$ RDF + Azospirillum + PSB + Boron @ $10 \mathrm{Kg} \mathrm{ha}^{-1}$. Meager information was available regarding the performance of integrated application of organics and micronutrient on brinjal in the experimental location. The present study may enlighten this unexplored section of nutrient management in brinjal.
\end{abstract}

Keywords: Azospirillum, Borax, Brinjal, PSB, Integrated Nutrient Management

\section{INTRODUCTION}

India is regarded as a horticultural paradise (Saravaiya and Patel, 2005), with a vast array of vegetables being cultivated in our country, brinjal is considered as one of the leading and the second major vegetable crops next to tomato. Brinjal (Solanum melongena) is also called as eggplant, a popular vegetable crop and is native to India (Kiran et al., 2010). India contributes about $28 \%$ of the total world production (Daunay et al., 2001). It is highly productive and usually finds a place as "poor man's crop". Purple fruits have higher amino acid content. Brinjal fruits have medicinal properties (Rajan and Markose, 2002). Some medicinal use of eggplant tissues and extract include treatment of diabetes, asthama, cholera, bronchitis and diarrhea, its fruit and leaves are reported to lower certain levels of blood cholesterol. The growth, yield and fruit quality of brinjal are largely dependent on a number of interacting factors.
Egg plant is a long duration crop with high yield which removes large quantities of nutrients from the soil. An egg-plant crop yielding $60 \mathrm{t} \mathrm{ha}^{-1}$ of fruit removes 190 $\mathrm{kg} \mathrm{N}, 10.9 \mathrm{~kg} \mathrm{P}$ and $128 \mathrm{~kg} \mathrm{~K}$ from soil (Hedge, 1997). Now-a-days demand for brinjal as a vegetable is increasing rapidly among the vegetable consumers in view of its better fruit color, size and taste. Average productivity of brinjal crop is quite low and there exists a good scope to improve its average productivity in India to fulfill both domestic and national needs.

The productivity of brinjal can be increased by using several techniques viz., organic farming, integrated nutrient management and good hybrid seeds. Since the nutrient turnover in soil plant system is considerably high in intensive vegetable cultivation, neither the chemical fertilizer nor the organic manure alone can help achieve sustainable production (Khan et al., 2008). Moreover, the application of high input technologies such as chemical fertilizers, pesticides, herbicides improve the production but there is growing 
concern over the adverse effects of the use of chemicals on human health, soil productivity and environment quality.(Sharma et al., 2012). Bio fertilizers improve the quantitative and qualitative features of many plants (Yousefi et al., 2011). In addition, biofertilizers stimulate plant growth, improve both soil structure and conditions, restore natural soil fertility and provide protection against drought and some soil borne diseases (Bashan et al., 2004). On the other hand, application of organics improves the soil physical, chemical and biological properties and has direct impact on moisture retention, root growth and nutrient conservation etc. (Kumar et al., 2011). Many countries have already introduced the organic production system with specific logo to provide individuality to the organic products in commercial trade (Sharma, 2011).

Application of NPK, bio-fertilizers like Azospirillum and phosphate solubilizing bacteria (PSB) with micronutrients viz., ferrous sulphate, zinc sulphate and boron spray bring profound changes in various metabolic processes within the plant system, thereby influencing plant growth and yield considerably. In recent years, importance of combined use of these inorganic fertilizers, biofertilizers and micronutrients is being realized particularly in brinjal to boost up plant growth, productivity and also on seed quality. The application of high input technologies such as chemical fertilizers, pesticides, herbicides improve the production but there is growing concern over the adverse effects of the use of chemicals on human health, soil productivity and environment quality. The integrated management of nutrient, in its proper perspective, may be adopted to support enhanced productivity and quality of vegetables (Kiran et al., 2010). Keeping in view the present investigation was carried out to study the effect of integrated nutrient management on the nutrient status and uptake of brinjal (Solanum melongena L.) in an inceptisol of West Bengal.

\section{MATERIALS AND METHODS}

Characterization of experimental site: Field trials were conducted in the new alluvial zone (Inceptisol) of Central Research Farm of Bidhan Chandra Krishi Viswavidyalaya at Gayeshpur Nadia district of West Bengal, India. The field is situated at in $22^{0} 58^{\prime} 162^{\prime \prime} \mathrm{N}$ latitude, $88^{0} 30^{\prime} 651^{\prime \prime} \mathrm{E}$ longitude, experiencing hot and humid climate with mean annual rainfall of $1310 \mathrm{~mm}$, minimum and maximum temperature of $21 \pm 1$ and 32 $\pm 2^{\circ} \mathrm{C}$, respectively. The soil was typic endoaquepts with silty clayey loamy texture, slightly alkaline in reaction. The experimental soils were non-saline (EC $0.278 \mathrm{dS} / \mathrm{m})$, sandy-clay loam in texture, neutral in reaction ( $\mathrm{pH} 6.7)$, low in organic carbon $(4.5 \mathrm{~g} / \mathrm{kg})$, available $\mathrm{N}$ (178 kg/ha), high in available P (51 kg/ha) and medium in available $\mathrm{K}$ contents $(137 \mathrm{~kg} / \mathrm{ha})$. The soils were high in DTPA extractable Fe $(25.3 \mathrm{~kg} / \mathrm{ha})$ and low in DTPA extractable $\mathrm{Zn}$ and B (4.56 and 0.43 $\mathrm{kg} / \mathrm{ha}$, respectively).

Experimental details: The experiment was laid out in two factor factorial randomized block design with each treatment being replicated three times and with

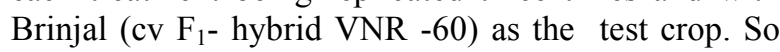
there were $48(4 \times 4 \times 3)$ plots $($ size $5 \mathrm{~m} \times 2 \mathrm{~m})$. The experiment consisted of sixteen treatments combinations comprising of four levels of fertilizers and biofertilizers viz; $\mathrm{F}_{1}=$ recommended dose of fertilizer $(\mathrm{RDF}) \mathrm{N}: \mathrm{P}: \mathrm{K}:$ : 125:100:50kg ha ${ }^{-1}, \mathrm{~F}_{2}=75 \% \mathrm{RDF}+$ Azospirillum (root dipping)@250 $\mathrm{g} \mathrm{ha}^{-1}, \mathrm{~F}_{3}=75 \% \mathrm{RDF}+\mathrm{PSB}$ (root dipping)@250 g ha ${ }^{-1}, \mathrm{~F}_{4}=75 \% \mathrm{RDF}+$ Azospirillum + PSB (root dipping) each @ $125 \mathrm{~g} \mathrm{ha}^{-1}$ and four levels of micronutrients viz; $\mathrm{S}_{1}=$ control, $\mathrm{S}_{2}=\mathrm{ZnSO}_{4} @ 25 \mathrm{~kg}$ $\mathrm{ha}^{-1}$ (Soil application), $\mathrm{S}_{3}=\mathrm{FeSO}_{4} @ 50 \mathrm{~kg} \mathrm{ha}^{-1}$ (Soil application) and $\mathrm{S}_{4}=$ Borax @ $10 \mathrm{~kg} \mathrm{ha}^{-1}$ (Soil application).

The recommended dose of inorganic fertilizers @ $125: 100: 50 \quad(\mathrm{~N}: \mathrm{P}: \mathrm{K}) \mathrm{kg} \mathrm{ha}{ }^{-1}$ were applied in the experimental plot. The entire amount of phosphorus (SSP), potassic fertilizer (MOP) and half of total nitrogenous fertilizer (urea) were applied at final land preparation. The rest half of the nitrogenous fertilizers were applied in two equal split doses (one quarter each) at 20-25 DAS and at 40-45 DAS respectively. Bio-fertilizers like Azospirillum and PSB@250 g ha ${ }^{-1}$ and Azospirillum + PSB each @125 $\mathrm{g} \mathrm{ha}^{-1}$ were applied in the selected plots as root dip treatment before transplanting of brinjal seedling. Micronutrient fertilizers were applied as soil application (SA) 4 days before transplanting of brinjal seedling. Fruits were harvested at their edible maturity stage i.e. when they are not fully ripened and have a bright purple colour and harvested at 3 days interval at peak fruiting stage.

Analytical methodologies: The methods involved in analyses of soil (both initial and post-harvest) and plant samples are depicted in Table 1.

Statistical interpretation: The experimental layout was set to be a $4 \times 4$ factorial design with three replications. Statistical conclusions were drawn by factorial analysis of the database. All statistical computations were done through SPSS V.16.0.

\section{RESULTS AND DISCUSSION}

Nutrient uptake in brinjal: The nutrients status of brinjal fruit, leaf and shoot (estimated in dry weight basis) obtained through different systems of integrated management have been recorded in tables 2 and $3 . \mathrm{N}$ accumulations ( $\mathrm{g} \mathrm{kg}^{-1}$ dry weight basis) in brinjal leaf, fruit and shoot were observed to vary within a range of 17.40, 16.36 and 9.47 (control) to 25.33, 23.72 and 15.77 (75\% RDF+ PSB+ Borax @ $10 \mathrm{~kg} \mathrm{ha}^{-1}$ ) respectively. The changes in the fruit protein content followed the same trend as observed in total $\mathrm{N}$ content. $\mathrm{P}$ accumulation ( $\mathrm{g} \mathrm{kg}^{-1}$ dry weight basis) in brinjal leaf, fruit and shoot were observed to vary within a range of $3.95,3.81$ and 3.65 (control) to $8.83,7.00$ and 8.16 (75\% RDF+ PSB+ Borax @ $10 \mathrm{~kg} \mathrm{ha}^{-1}$ ), respectively. Potassium accumulations ( $\mathrm{g} \mathrm{kg}^{-1}$ dry weight) in brinjal 
Table 1. Analytical methodologies parameters.

\begin{tabular}{|c|c|c|c|}
\hline Parameter & Methodology & Citation & Equipment used \\
\hline \multicolumn{4}{|l|}{ Soil analyses } \\
\hline Sand-Silt-Clay & Hydrometer method & Bouyoucos (1962) & Hydrometer \\
\hline $\mathrm{pH}$ & (in $1: 2.5::$ Soil : Water) & Jackson (1967) & \multirow{2}{*}{ m-processor based $\mathrm{pH}$-EC-Ion meter } \\
\hline $\mathrm{EC}$ & (in $1: 2.5::$ Soil : Water) & Jackson (1967) & \\
\hline Organic carbon & Wet oxidation method & Jackson (1973) & - \\
\hline Available N & Hot alkaline $\mathrm{KMnO}_{4}$ Method & Subbiah and Asija (1956) & Kjeldahl apparatus \\
\hline Available P & $0.5 \mathrm{M} \mathrm{NaHCO}_{3}$ extraction & Olsen et al.(1954) & Spectrophotometer \\
\hline Available K & Neutral $\mathrm{N} \mathrm{NH}_{4} \mathrm{OAc}$ extraction & Brown and Warncke (1988) & Flame photometer \\
\hline Available Fe & DTPA extraction & Lindsay and Norvell (1978) & Atomic Absorption Spectrophotometer \\
\hline Available $\mathrm{Zn}$ & DTPA extraction & Lindsay and Norvell (1978) & Atomic Absorption Spectrophotometer \\
\hline Available B & Hot water extraction & Berger and Truog (1939) & Spectrophotometer \\
\hline \multicolumn{4}{|c|}{ Analyses of plant samples } \\
\hline Total N & Concentrated $\mathrm{H}_{2} \mathrm{SO}_{4}$ digestion & AOAC, 1995 & Micro-Kjeldahl's method \\
\hline Protein percentage & $\begin{array}{l}\text { Accounted by multiplying } \\
\text { total nitrogen content by } 6.25\end{array}$ & $\begin{array}{l}\text { Sadasivam and Manickam, } \\
1996\end{array}$ & - \\
\hline Total P & $\begin{array}{l}\text { Tri-acid mixture } \\
\left(\mathrm{HNO}_{3}: \mathrm{H}_{2} \mathrm{SO}_{4}: \mathrm{HClO}_{4}:: 9: 1: 4\right) \\
\text { digestion }\end{array}$ & Jackson, 1973 & Spectrophotometer \\
\hline Total K & $\begin{array}{l}\text { Tri-acid mixture } \\
\left(\mathrm{HNO}_{3}: \mathrm{H}_{2} \mathrm{SO}_{4}: \mathrm{HClO}_{4}:: 9: 1: 4\right) \\
\text { digestion }\end{array}$ & Jackson, 1973 & Flame photometer \\
\hline Total $\mathrm{Fe}$ and $\mathrm{Zn}$ & $\begin{array}{l}\text { Tri-acid mixture } \\
\left(\mathrm{HNO}_{3}: \mathrm{H}_{2} \mathrm{SO}_{4}: \mathrm{HClO}_{4}:: 9: 1: 4\right) \\
\text { digestion }\end{array}$ & Jackson, 1973 & Atomic Absorption Spectrophotometer \\
\hline Total B & Dry ashing & Gaines and Mitchell (1979) & Spectrophotometer \\
\hline
\end{tabular}

leaf, fruit and shoot were observed to vary within a range of $17.03,15.14$ and 11.42 (control) to 31.64 , 23.81 and 16.60 (75\% RDF + Azospirillum + PSB + Borax@10 kg ha ${ }^{-1}$ ). Maximum concentration of N, P and $\mathrm{K}$ in leaf, shoot and fruit was observed in $75 \%$ RDF + Azospirillum + PSB + Borax @ $10 \mathrm{~kg} \mathrm{ha}^{-1}$ treatment. Our observations in this regard have been substantiated by findings of several earlier workers like Srijaya and Sitaramayya (2006) who found highest nitrogen (2.28\%), $\mathrm{P}(0.224 \%), \mathrm{K}(1.17 \%)$ against treatment RDF + gypsum and $\mathrm{RDF}+\mathrm{Zn}$ (soil application), respectively. The range of $\mathrm{N}$ was 1.92 to $2.10 \%, 0.122$ to $0.244 \%$ for $\mathrm{P}$ and 1.10 to $1.17 \%$ for $\mathrm{K}$ in plant while in fruits the $\mathrm{P}$ and $\mathrm{K}$ concentrations were more than $\mathrm{N}$ as compared to plant. Patil and Patil (2000) recorded N, P and $\mathrm{K}$ contents in different cultivars of brinjal fruits which ranged from 2.04 to $3.26,0.35$ to 0.55 and 1.50 to 3.89 per cent, respectively.

Positive impact of biofertilizers on nutrient uptake and quality of vegetables has already been reported (Choudhury et al., 2005; Kadlag et al., 2007). Singh et al., (2004) also reported that the integrated use of organic and inorganic sources of nutrients and biofertilizers increased the $\mathrm{N}, \mathrm{P}$ and $\mathrm{K}$ concentration in the plants (including fruits of okra, pea and tomato under cropping system in a Mollisols while higher higher protein and ascorbic acid in cauliflower with $60 \mathrm{~kg} \mathrm{~N}$ and Azotobacter has been reported by Bashyal (2011). Iron accumulations ( $\mathrm{g} \mathrm{kg}^{-1}$ dry weight) in brinjal leaf, fruit and shoot were observed to vary within a range of 121.87, 125.67, 124.67 (control) to 316.72 (75\% RDF + PSB + $\left.\mathrm{FeSO}_{4} @ 50 \mathrm{~kg} \mathrm{ha}{ }^{-1}\right), 265.83(75 \% \mathrm{RDF}+$ Azospirillum + $\left.\mathrm{FeSO}_{4} @ 50 \mathrm{~kg} \mathrm{ha}^{-1}\right), 251.50(75 \%$ $\left.\mathrm{RDF}+\mathrm{Azo}+\mathrm{PSB}+\mathrm{FeSO}_{4} @ 50 \mathrm{~kg} \mathrm{ha}{ }^{-1}\right)$, respectively. Zinc accumulations ( $\mathrm{g} \mathrm{kg}^{-1}$ dry weight) in brinjal leaf, fruit and shoot were observed to vary within a range of $25.50,25.43$ and 25.00 (control) to 43.10, 42.60 and $39.86(75 \% \mathrm{RDF}+$ Azospirillum $+\mathrm{PSB}+$ $\mathrm{ZnSO}_{4} @ 25 \mathrm{~kg} \mathrm{ha}^{-1}$ ), respectively. Boron accumulations ( $\mathrm{g} \mathrm{kg}^{-1}$ dry weight) in brinjal leaf, fruit and shoot were observed to vary within a range $0.70,0.34$ and 0.27 (control) to $1.19,0.51$ and $0.46(75 \% \mathrm{RDF}+$ Azospirillum + PSB + Borax@10 kg ha $\left.{ }^{-1}\right)$, respectively. Integrated nutrient management and plant uptake or accumulation of micronutrient elements have been studied by several workers in a no. of crops and cropping system. Working with brinjal cv. "CVK" in Coimbatore, Selvi et al., (2004) recorded that a $\mathrm{Zn}$ uptake ranging from 278 to $493 \mathrm{~g} / \mathrm{ha}$. They further noticed that the content of zinc in Brinjal fruits ranged from 42.8 to $154.4 \mathrm{ppm}$. Selvi et al. (2004) recorded that Fe uptake ranged from 2.16 to $3.49 \mathrm{~kg} / \mathrm{ha}$ as well as iron content in brinjal fruit ranged from $313-401$ ppm. Singh et al., (2012) reported that $\mathrm{Zn}$ content in 
Table.2: Nutrient accumulation (dry weight recoveries) in brinjal plant.

\begin{tabular}{|c|c|c|c|c|c|c|c|c|c|c|}
\hline \multirow{2}{*}{ Treatment } & \multirow{2}{*}{$\begin{array}{l}\text { Protein } \\
(\%)\end{array}$} & \multicolumn{3}{|c|}{$\mathrm{N}$ content $\left(\mathrm{g} \mathrm{kg}^{-1}\right)$} & \multicolumn{3}{|c|}{$P$ content $\left(\mathrm{g} \mathrm{kg}^{-1}\right)$} & \multicolumn{3}{|c|}{ K content $\left(\mathrm{g} \mathrm{kg}^{-1}\right)$} \\
\hline & & Leaf & Fruit & Shoot & Leaf & Fruit & Shoot & Leaf & Fruit & Shoot \\
\hline \multicolumn{11}{|c|}{ Fertilizers and Bio-fertilizers } \\
\hline $\mathrm{F}_{1}$ & $10.68^{\mathrm{b}}$ & $20.02^{\mathrm{b}}$ & $17.09^{\mathrm{b}}$ & $16.90^{\mathrm{b}}$ & $5.69^{d}$ & $5.38^{\mathrm{d}}$ & $5.29^{\mathrm{d}}$ & $20.69^{b}$ & $16.90^{\mathrm{b}}$ & $11.84^{\mathrm{b}}$ \\
\hline $\mathrm{F}_{2}$ & $11.89^{\mathrm{ab}}$ & $22.51^{\mathrm{a}}$ & $19.02^{\mathrm{ab}}$ & $19.13^{\mathrm{ab}}$ & $6.65^{\mathrm{c}}$ & $5.76^{\mathrm{c}}$ & $6.30^{\mathrm{c}}$ & $23.84^{\mathrm{ab}}$ & $19.13^{\mathrm{ab}}$ & $14.04^{\mathrm{a}}$ \\
\hline $\mathrm{F}_{3}$ & $13.34^{\mathrm{a}}$ & $23.94^{\mathrm{a}}$ & $21.34^{\mathrm{a}}$ & $18.80^{\mathrm{ab}}$ & $8.00^{\mathrm{a}}$ & $6.52^{\mathrm{a}}$ & $7.49^{\mathrm{a}}$ & $23.11^{\mathrm{ab}}$ & $18.80^{\mathrm{ab}}$ & $13.11^{\mathrm{ab}}$ \\
\hline $\mathrm{F}_{4}$ & $12.71^{\mathrm{a}}$ & $23.98^{\mathrm{a}}$ & $20.34^{\mathrm{a}}$ & $20.33^{\mathrm{a}}$ & $7.69^{\mathrm{b}}$ & $6.47^{\mathrm{b}}$ & $7.12^{\mathrm{b}}$ & $25.96^{\mathrm{a}}$ & $20.33^{\mathrm{a}}$ & $14.58^{\mathrm{a}}$ \\
\hline \multicolumn{11}{|c|}{ Micronutrients } \\
\hline $\mathrm{S}_{1}$ & $11.25^{\mathrm{a}}$ & $21.75^{\mathrm{a}}$ & $18.00^{\mathrm{a}}$ & $17.26^{\mathrm{a}}$ & $6.22^{\mathrm{c}}$ & $5.37^{\mathrm{c}}$ & $5.89^{d}$ & $20.78^{\mathrm{b}}$ & $17.26^{\mathrm{a}}$ & $12.92^{\mathrm{a}}$ \\
\hline $\mathrm{S}_{2}$ & $12.54^{\mathrm{a}}$ & $23.28^{\mathrm{a}}$ & $20.06^{\mathrm{a}}$ & $19.12^{\mathrm{a}}$ & $7.08^{\mathrm{b}}$ & $6.15^{\mathrm{b}}$ & $6.24^{\mathrm{c}}$ & $23.19^{\mathrm{ab}}$ & $19.12^{\mathrm{a}}$ & $13.21^{\mathrm{a}}$ \\
\hline $\mathrm{S}_{3}$ & $12.56^{\mathrm{a}}$ & $22.94^{\mathrm{a}}$ & $20.10^{\mathrm{a}}$ & $18.94^{\mathrm{a}}$ & $7.11^{\mathrm{b}}$ & $6.13^{\mathrm{b}}$ & $6.85^{\mathrm{b}}$ & $25.36^{\mathrm{a}}$ & $18.94^{\mathrm{a}}$ & $13.17 \mathrm{a}$ \\
\hline $\mathrm{S}_{4}$ & $12.27^{\mathrm{a}}$ & $22.49^{\mathrm{a}}$ & $19.63^{\mathrm{a}}$ & $19.84^{\mathrm{a}}$ & $7.62^{\mathrm{a}}$ & $6.48^{\mathrm{a}}$ & $7.23^{\mathrm{a}}$ & $24.27^{\mathrm{ab}}$ & $19.84^{\mathrm{a}}$ & $14.27^{\mathrm{a}}$ \\
\hline \multicolumn{11}{|c|}{ Interaction (F X S) } \\
\hline$\overline{F_{1} S_{1}}$ & $10.23^{\mathrm{Dc}}$ & $17.40^{\mathrm{b}}$ & $16.36^{\mathrm{bc}}$ & $15.14^{\mathrm{b}}$ & $3.95^{\mathrm{g}}$ & $3.81^{\mathrm{h}}$ & $3.65^{\mathrm{g}}$ & $17.03^{\mathrm{c}}$ & $15.14^{\mathrm{b}}$ & $11.42^{\mathrm{b}}$ \\
\hline $\mathrm{F}_{1} \mathrm{~S}_{2}$ & $10.40^{\mathrm{bc}}$ & $21.48^{\mathrm{ab}}$ & $16.64^{\mathrm{bc}}$ & $17.64^{\mathrm{ab}}$ & $6.06^{\mathrm{f}}$ & $6.05^{\mathrm{e}}$ & $5.45^{\mathrm{f}}$ & $21.52^{\mathrm{abc}}$ & $17.64^{\mathrm{ab}}$ & $11.79^{b}$ \\
\hline $\mathrm{F}_{1} \mathrm{~S}_{3}$ & $11.75^{\mathrm{abc}}$ & $20.49^{\mathrm{ab}}$ & $18.80^{\mathrm{abc}}$ & $17.47^{\mathrm{ab}}$ & $6.29^{\mathrm{ef}}$ & $5.43^{\mathrm{g}}$ & $5.93^{\mathrm{e}}$ & $22.74^{\mathrm{abc}}$ & $17.47^{\mathrm{ab}}$ & $11.68^{\mathrm{b}}$ \\
\hline $\mathrm{F}_{1} \mathrm{~S}_{4}$ & $10.35^{\mathrm{bc}}$ & $20.70^{\mathrm{ab}}$ & $16.57^{\mathrm{bc}}$ & $17.35^{\mathrm{ab}}$ & $6.48^{\mathrm{def}}$ & $6.22^{\mathrm{d}}$ & $6.12^{\mathrm{de}}$ & $21.46^{\mathrm{abc}}$ & $17.35^{\mathrm{ab}}$ & $12.45^{\mathrm{ab}}$ \\
\hline $\mathrm{F}_{2} \mathrm{~S}_{1}$ & $11.66^{\mathrm{abc}}$ & $21.88^{\mathrm{ab}}$ & $18.66^{\mathrm{abc}}$ & $17.92^{\mathrm{ab}}$ & $5.99^{\mathrm{f}}$ & $5.44^{\mathrm{g}}$ & $6.15^{\mathrm{de}}$ & $23.18^{\mathrm{abc}}$ & $17.92^{\mathrm{ab}}$ & $13.73 \mathrm{ab}$ \\
\hline $\mathrm{F}_{2} \mathrm{~S}_{2}$ & $11.92^{\mathrm{abc}}$ & $23.58^{\mathrm{ab}}$ & $19.07^{\mathrm{abc}}$ & $19.61^{\mathrm{ab}}$ & $6.84^{\text {cde }}$ & $5.66^{\mathrm{f}}$ & $6.17^{\mathrm{de}}$ & $24.43^{\mathrm{abc}}$ & $19.61^{\mathrm{ab}}$ & $14.10 \mathrm{ab}$ \\
\hline $\mathrm{F}_{2} \mathrm{~S}_{3}$ & $11.92^{\mathrm{abc}}$ & $23.66^{\mathrm{ab}}$ & $19.08^{\mathrm{abc}}$ & $19.54^{\mathrm{ab}}$ & $6.92^{\mathrm{cd}}$ & $6.20^{\mathrm{d}}$ & $6.35^{\mathrm{de}}$ & $27.25^{\mathrm{abc}}$ & $19.54^{\mathrm{ab}}$ & $13.76 \mathrm{ab}$ \\
\hline $\mathrm{F}_{2} \mathrm{~S}_{4}$ & $12.04^{\mathrm{abc}}$ & $20.92^{\mathrm{ab}}$ & $19.26^{\mathrm{abc}}$ & $19.47^{\mathrm{ab}}$ & $6.86^{\text {cde }}$ & $5.75^{\mathrm{f}}$ & $6.53^{\mathrm{cd}}$ & $20.49^{\mathrm{bc}}$ & $19.47^{\mathrm{ab}}$ & $14.58 \mathrm{ab}$ \\
\hline $\mathrm{F}_{3} \mathrm{~S}_{1}$ & $13.14^{\mathrm{abc}}$ & $23.64^{\mathrm{ab}}$ & $21.01^{\mathrm{abc}}$ & $18.19^{\mathrm{ab}}$ & $7.53^{\mathrm{b}}$ & $6.08^{\mathrm{e}}$ & $6.93^{\mathrm{bc}}$ & $21.32^{\mathrm{abc}}$ & $18.19^{\mathrm{ab}}$ & $12.59 \mathrm{ab}$ \\
\hline $\mathrm{F}_{3} \mathrm{~S}_{2}$ & $14.53^{\mathrm{ab}}$ & $24.80^{\mathrm{a}}$ & $23.24^{\mathrm{ab}}$ & $19.23^{\mathrm{ab}}$ & $8.27^{\mathrm{a}}$ & $6.65^{\mathrm{b}}$ & $7.03^{\mathrm{b}}$ & $23.88^{\mathrm{abc}}$ & $19.23^{\mathrm{ab}}$ & $13.54 \mathrm{ab}$ \\
\hline $\mathrm{F}_{3} \mathrm{~S}_{3}$ & $13.84^{\mathrm{abc}}$ & $24.32^{\mathrm{a}}$ & $22.16^{\mathrm{abc}}$ & $19.05^{\mathrm{ab}}$ & $7.58^{\mathrm{b}}$ & $6.36^{\mathrm{c}}$ & $7.85^{\mathrm{a}}$ & $23.76^{\mathrm{abc}}$ & $19.05^{\mathrm{ab}}$ & $12.86 \mathrm{ab}$ \\
\hline $\mathrm{F}_{3} \mathrm{~S}_{4}$ & $11.85^{\mathrm{abc}}$ & $23.00^{\mathrm{ab}}$ & $18.97^{\mathrm{abc}}$ & $19.71^{\mathrm{ab}}$ & $8.63^{\mathrm{a}}$ & $7.00^{\mathrm{a}}$ & $8.16^{\mathrm{a}}$ & $23.48^{\mathrm{abc}}$ & $18.71^{\mathrm{ab}}$ & $13.46 \mathrm{ab}$ \\
\hline $\mathrm{F}_{4} \mathrm{~S}_{1}$ & $9.97^{\mathrm{c}}$ & $24.07^{\mathrm{a}}$ & $15.95^{\mathrm{c}}$ & $17.80^{\mathrm{ab}}$ & $7.42^{\mathrm{bc}}$ & $6.15^{\mathrm{de}}$ & $6.82^{\mathrm{bc}}$ & $21.58^{\mathrm{abc}}$ & $17.80^{\mathrm{ab}}$ & $13.93 \mathrm{ab}$ \\
\hline $\mathrm{F}_{4} \mathrm{~S}_{2}$ & $13.32^{\mathrm{abc}}$ & $23.26^{\mathrm{ab}}$ & $21.31^{\mathrm{abc}}$ & $20.00^{\mathrm{ab}}$ & $7.16^{\mathrm{bc}}$ & $6.24^{\mathrm{d}}$ & $6.29^{\mathrm{de}}$ & $22.94^{\mathrm{abc}}$ & $20.00^{\mathrm{ab}}$ & $13.41 \mathrm{ab}$ \\
\hline $\mathrm{F}_{4} \mathrm{~S}_{3}$ & $12.74^{\mathrm{abc}}$ & $23.27^{\mathrm{ab}}$ & $20.38^{\mathrm{abc}}$ & $19.69^{\mathrm{ab}}$ & $7.64^{b}$ & $6.54^{\mathrm{b}}$ & $7.26^{\mathrm{b}}$ & $27.68^{\mathrm{ab}}$ & $19.69^{\mathrm{ab}}$ & $14.39^{\mathrm{ab}}$ \\
\hline $\mathrm{F}_{4} \mathrm{~S}_{4}$ & $14.83^{\mathrm{a}}$ & $25.33^{\mathrm{a}}$ & $23.72^{\mathrm{a}}$ & $23.81^{\mathrm{a}}$ & $8.52^{\mathrm{a}}$ & $6.93^{\mathrm{a}}$ & $8.09^{\mathrm{b}}$ & $31.64^{\mathrm{a}}$ & $23.81^{\mathrm{a}}$ & $16.60^{\mathrm{a}}$ \\
\hline
\end{tabular}

$\mathrm{F}_{1}-125: 100: 50$ (RDF), $\mathrm{F}_{2}-75 \% \mathrm{RDF}+$ Azotobacter, $\mathrm{F}_{3}-75 \% \mathrm{RDF}+\mathrm{PSB}, \mathrm{F}_{4}-75 \% \mathrm{RDF}+$ Azotobacter + PSB; $\mathrm{S}_{1}-\mathrm{Control}, \mathrm{S}_{2}-$ $\mathrm{ZnSO}_{4} @ 25 \mathrm{~kg} / \mathrm{ha}, \mathrm{S}_{3}-\mathrm{FeSO}_{4} @ 50 \mathrm{~kg} / \mathrm{ha}, \mathrm{S}_{4}-$ Borax @10 kg/ha; a, b different superscriptions against means denote significant differences (otherwise statistically at par) at $\mathrm{P}<0.05$ by Tukey's Honest significant Difference Test

brinjal stem, leaf and fruits to the tune of 87,102 and 29 (kg/g dry wt.), respectively. Karuppaiah (2005) stated that foliar application of borax $(0.5 \%)$ at 35,50 and 65 DAT was found to be best in terms of no. of flowers per plant, no. of fruits per plant.

Nutrient status of post-harvest soil of brinjal: The organic matter and nutrient status of post harvest soil of brinjal obtained under different INM treatments as recorded. The oxidizable organic carbon content $(\mathrm{g} \mathrm{kg}$ ${ }^{1}$ ) of post harvest soil of brinjal has been observed to vary within a range of 4.46 (control) to $8.049(75 \%$ RDF+ Azospirillum + PSB + Borax@10 kg ha ${ }^{-1}$ ). Since chemical NPK could not, any way, contribute to soil organic fraction, it is presumably the bacterial fertilizers which contributed to increased oxidizable organic C pool through greater mineralization of soil organic matter by enhanced microbial population and in such the performance of combined addition of
Azospirillum + PSB remained most pronounced (Table 4).

Total nitrogen $\left(\mathrm{g} \mathrm{kg}^{-1}\right)$ of post harvest soil of brinjal has been observed to vary within a range of 0.49 (control) to 1.05 (75\% RDF + Azospirillum + PSB + Borax@10 kg ha-1) (Table 4). The significant increase in total $\mathrm{N}$ of post harvest soils receiving NPK and Azospirillum remained quite imperative due to further $\mathrm{N}$ addition through biological fixation. Ladha $e t$ al., (2014) reported that the increase in the nitrogen content of the post-harvest soil sample treated with biofertilizer might be due to the release of more of nitrogenous substance in the soil. Application of micronutrient fertilizers also observed to increase total soil $\mathrm{N}$ significantly over the control counterparts for reasons could not be explained through available findings obtained from the present investigation. Available nitrogen $\mathrm{kg} \mathrm{ha}^{-1}$ have been ranged from 
Table 3. Micronutrient accumulation (dry weight recoveries) in brinjal plant.

\begin{tabular}{|c|c|c|c|c|c|c|c|c|c|}
\hline \multirow{2}{*}{ Treatment } & \multicolumn{3}{|c|}{ Fe content $\left(\mathrm{g} \mathrm{kg}^{-1}\right)$} & \multicolumn{3}{|c|}{$\mathrm{Zn}$ content $\left(\mathrm{g} \mathrm{kg}^{-1}\right)$} & \multicolumn{3}{|c|}{ B content $\left(\mathrm{g} \mathrm{kg}^{-1}\right)$} \\
\hline & Leaf & Fruit & Shoot & Leaf & Fruit & Shoot & Leaf & Fruit & Shoot \\
\hline \multicolumn{10}{|c|}{ Fertilizers and Bio-fertilizers } \\
\hline$\overline{F_{1}}$ & $142.03^{\mathrm{c}}$ & $143.57^{\mathrm{c}}$ & $152.67^{\mathrm{b}}$ & $27.51^{b}$ & $30.40^{\mathrm{c}}$ & $29.84^{\mathrm{c}}$ & $0.73^{b}$ & $0.37^{\mathrm{a}}$ & $0.33^{\mathrm{a}}$ \\
\hline $\mathrm{F}_{2}$ & $210.45^{\mathrm{ab}}$ & $166.54^{\mathrm{b}}$ & $210.78^{\mathrm{a}}$ & $33.32^{\mathrm{a}}$ & $32.73^{\mathrm{bc}}$ & $32.41^{\mathrm{bc}}$ & $0.95^{\mathrm{a}}$ & $0.41^{\mathrm{a}}$ & $0.34^{\mathrm{a}}$ \\
\hline $\mathrm{F}_{3}$ & $229.52^{\mathrm{a}}$ & $188.88^{\mathrm{a}}$ & $201.75^{\mathrm{a}}$ & $34.25^{\mathrm{a}}$ & $35.40^{\mathrm{ab}}$ & $34.40^{\mathrm{ab}}$ & $0.91^{\mathrm{a}}$ & $0.42 \mathrm{a}$ & $0.35^{\mathrm{a}}$ \\
\hline $\mathrm{F}_{4}$ & $189.26^{\mathrm{b}}$ & $197.54^{\mathrm{a}}$ & $201.61^{\mathrm{a}}$ & $37.33^{\mathrm{a}}$ & $38.99^{\mathrm{a}}$ & $37.05^{\mathrm{a}}$ & $1.04^{\mathrm{a}}$ & $0.45^{\mathrm{a}}$ & $0.35^{\mathrm{a}}$ \\
\hline \multicolumn{10}{|c|}{ Micronutrients } \\
\hline $\mathrm{S}_{1}$ & $160.37^{b}$ & $153.63^{b}$ & $164.44^{b}$ & $30.30^{b}$ & $28.57 \mathrm{c}$ & $28.83^{b}$ & $0.83^{\mathrm{b}}$ & $0.39^{\mathrm{ab}}$ & $0.31^{b}$ \\
\hline $\mathrm{S}_{2}$ & $183.10^{\mathrm{b}}$ & $160.08^{\mathrm{b}}$ & $193.75^{\mathrm{ab}}$ & $37.71^{\mathrm{a}}$ & $38.76^{\mathrm{a}}$ & $37.19^{\mathrm{a}}$ & $0.91^{\mathrm{ab}}$ & $0.40^{\mathrm{ab}}$ & $0.33^{\mathrm{b}}$ \\
\hline $\mathrm{S}_{3}$ & $249.00^{\mathrm{a}}$ & $219.50^{\mathrm{a}}$ & $236.60^{\mathrm{a}}$ & $33.06^{\mathrm{ab}}$ & $35.27^{\mathrm{ab}}$ & $33.60^{\mathrm{a}}$ & $0.92^{\mathrm{ab}}$ & $0.39^{\mathrm{b}}$ & $0.32^{\mathrm{b}}$ \\
\hline $\mathrm{S}_{4}$ & $178.78^{b}$ & $163.32^{b}$ & $172.02^{\mathrm{b}}$ & $31.34^{\mathrm{b}}$ & $34.92^{\mathrm{b}}$ & $34.07^{\mathrm{a}}$ & $0.99^{\mathrm{a}}$ & $0.47^{\mathrm{a}}$ & $0.40^{\mathrm{a}}$ \\
\hline \multicolumn{10}{|c|}{ Interaction (F X S) } \\
\hline$\overline{\mathrm{F}_{1} \mathrm{~S}_{1}}$ & $121.87^{\mathrm{e}}$ & $124.67^{\mathrm{g}}$ & $125.67^{\mathrm{c}}$ & $25.50^{\mathrm{b}}$ & $25.43^{\mathrm{e}}$ & $25.00^{\mathrm{e}}$ & $0.70^{\mathrm{b}}$ & $0.34^{\mathrm{a}}$ & $0.27^{\mathrm{d}}$ \\
\hline $\mathrm{F}_{1} \mathrm{~S}_{2}$ & $144.12^{\mathrm{e}}$ & $127.00^{\mathrm{g}}$ & $145.33^{\mathrm{abc}}$ & $31.43^{\mathrm{ab}}$ & $34.16^{\text {abcde }}$ & $34.59^{\text {abcde }}$ & $0.74^{\mathrm{b}}$ & $0.36^{\mathrm{a}}$ & $0.36^{\mathrm{abcd}}$ \\
\hline $\mathrm{F}_{1} \mathrm{~S}_{3}$ & $157.45^{\mathrm{e}}$ & $152.50^{\text {defg }}$ & $163.68^{\mathrm{abc}}$ & $26.33^{\mathrm{b}}$ & $30.13^{\text {cde }}$ & $28.58^{\text {cde }}$ & $0.77^{b}$ & $0.38^{\mathrm{a}}$ & $0.30^{\text {cd }}$ \\
\hline $\mathrm{F}_{1} \mathrm{~S}_{4}$ & $144.67^{\mathrm{e}}$ & $170.10^{\mathrm{cd}}$ & $176.00^{\mathrm{abc}}$ & $26.79^{b}$ & $31.88^{\text {bcde }}$ & $31.21^{\text {abcde }}$ & $0.72^{b}$ & $0.41^{\mathrm{a}}$ & $0.37^{\mathrm{abcd}}$ \\
\hline $\mathrm{F}_{2} \mathrm{~S}_{1}$ & $145.62 \mathrm{e}$ & $135.83^{\mathrm{fg}}$ & $141.60^{\mathrm{bc}}$ & $35.19^{\mathrm{ab}}$ & $30.50^{\text {cde }}$ & $31.83^{\text {abcde }}$ & $0.90^{\mathrm{ab}}$ & $0.40^{\mathrm{a}}$ & $0.35^{\mathrm{abcd}}$ \\
\hline $\mathrm{F}_{2} \mathrm{~S}_{2}$ & $251.82^{\mathrm{abc}}$ & $168.50^{\text {cde }}$ & $255.83^{\mathrm{ab}}$ & $38.93^{\mathrm{ab}}$ & $38.83^{\mathrm{abcd}}$ & $36.19^{\mathrm{abcd}}$ & $0.98^{\mathrm{ab}}$ & $0.38^{\mathrm{a}}$ & $0.32^{\mathrm{bcd}}$ \\
\hline $\mathrm{F}_{2} \mathrm{~S}_{3}$ & $265.68^{\mathrm{a}}$ & $224.67^{\mathrm{ab}}$ & $265.83^{\mathrm{a}}$ & $33.60^{\mathrm{ab}}$ & $32.77^{\text {abcde }}$ & $29.49^{\text {bcde }}$ & $0.93^{\mathrm{ab}}$ & $0.38^{\mathrm{a}}$ & $0.31^{\mathrm{bcd}}$ \\
\hline $\mathrm{F}_{2} \mathrm{~S}_{4}$ & $178.67^{\text {cde }}$ & $137.17^{\mathrm{efg}}$ & $179.83^{\mathrm{abc}}$ & $25.67^{\mathrm{b}}$ & $28.83^{\mathrm{de}}$ & $32.10^{\text {abcde }}$ & $1.00^{\mathrm{ab}}$ & $0.46^{\mathrm{a}}$ & $0.38^{\mathrm{abc}}$ \\
\hline $\mathrm{F}_{3} \mathrm{~S}_{1}$ & $189.55^{\text {bcde }}$ & $158.50^{\mathrm{def}}$ & $194.00^{\mathrm{abc}}$ & $29.33^{\mathrm{ab}}$ & $26.50^{\mathrm{e}}$ & $27.00^{\mathrm{de}}$ & $0.82^{\mathrm{ab}}$ & $0.40^{\mathrm{a}}$ & $0.32^{\mathrm{bcd}}$ \\
\hline $\mathrm{F}_{3} \mathrm{~S}_{2}$ & $165.17^{\mathrm{e}}$ & $141.33^{\text {defg }}$ & $165.17^{\mathrm{abc}}$ & $37.48^{\mathrm{ab}}$ & $39.46^{\mathrm{abc}}$ & $38.11^{\mathrm{abc}}$ & $0.82^{\mathrm{ab}}$ & $0.41^{\mathrm{a}}$ & $0.31^{\mathrm{bcd}}$ \\
\hline $\mathrm{F}_{3} \mathrm{~S}_{3}$ & $316.72^{\mathrm{a}}$ & $249.33^{\mathrm{a}}$ & $261.70^{\mathrm{ab}}$ & $35.74^{\mathrm{ab}}$ & $37.81^{\mathrm{abcd}}$ & $37.50^{\mathrm{abc}}$ & $0.96^{\mathrm{ab}}$ & $0.39^{\mathrm{a}}$ & $0.36^{\text {abcd }}$ \\
\hline $\mathrm{F}_{3} \mathrm{~S}_{4}$ & $246.67^{\mathrm{abcd}}$ & $206.33^{b}$ & $186.15^{\mathrm{abc}}$ & $34.44^{\mathrm{ab}}$ & $37.83^{\mathrm{abcd}}$ & $35.00^{\mathrm{abcd}}$ & $1.04^{\mathrm{ab}}$ & $0.48^{\mathrm{a}}$ & $0.41^{\mathrm{ab}}$ \\
\hline $\mathrm{F}_{4} \mathrm{~S}_{1}$ & $184.43^{\text {bcde }}$ & $195.50^{\mathrm{bc}}$ & $196.50^{\mathrm{abc}}$ & $31.17^{\mathrm{ab}}$ & $31.83^{\text {bcde }}$ & $31.50^{\text {abcde }}$ & $0.91^{\mathrm{ab}}$ & $0.42^{\mathrm{a}}$ & $0.32^{\mathrm{bcd}}$ \\
\hline $\mathrm{F}_{4} \mathrm{~S}_{2}$ & $171.32^{\mathrm{de}}$ & $203.50^{b}$ & $208.67^{\mathrm{abc}}$ & $43.10^{\mathrm{a}}$ & $42.60^{\mathrm{a}}$ & $39.86^{\mathrm{a}}$ & $1.08^{\mathrm{ab}}$ & $0.44^{\mathrm{a}}$ & $0.35^{\mathrm{abcd}}$ \\
\hline $\mathrm{F}_{4} \mathrm{~S}_{3}$ & $256.17^{\mathrm{ab}}$ & $251.50^{\mathrm{a}}$ & $255.18^{\mathrm{ab}}$ & $36.58^{\mathrm{ab}}$ & $40.37^{\mathrm{abc}}$ & $38.84^{\mathrm{ab}}$ & $1.00^{\mathrm{ab}}$ & $0.40^{\mathrm{a}}$ & $0.30^{\mathrm{cd}}$ \\
\hline $\mathrm{F}_{4} \mathrm{~S}_{4}$ & $145.13^{\mathrm{e}}$ & $139.67^{\text {defg }}$ & $146.08^{\mathrm{abc}}$ & $38.49^{\mathrm{ab}}$ & $41.14^{\mathrm{ab}}$ & $37.19^{\mathrm{abc}}$ & $1.19^{\mathrm{a}}$ & $0.51^{\mathrm{a}}$ & $0.45^{\mathrm{a}}$ \\
\hline
\end{tabular}

$\mathrm{F}_{1}-125: 100: 50(\mathrm{RDF}), \mathrm{F}_{2}-75 \% \mathrm{RDF}+$ Azotobacter, $\mathrm{F}_{3}-75 \% \mathrm{RDF}+\mathrm{PSB}, \mathrm{F}_{4}-75 \% \mathrm{RDF}+$ Azotobacter $+\mathrm{PSB} ; \mathrm{S}_{1}-\mathrm{Control}, \mathrm{S}_{2}-$ $\mathrm{ZnSO}_{4} @ 25 \mathrm{~kg} / \mathrm{ha}, \mathrm{S}_{3}-\mathrm{FeSO}_{4} @ 50 \mathrm{~kg} / \mathrm{ha}, \mathrm{S}_{4}-$ Borax @10 kg/ha; a, b different superscriptions against means denote significant differences (otherwise statistically at par) at $\mathrm{P}<0.05$ by Tukey's Honest significant Difference Test

Total yield ( $\mathrm{t} / \mathrm{ha})$

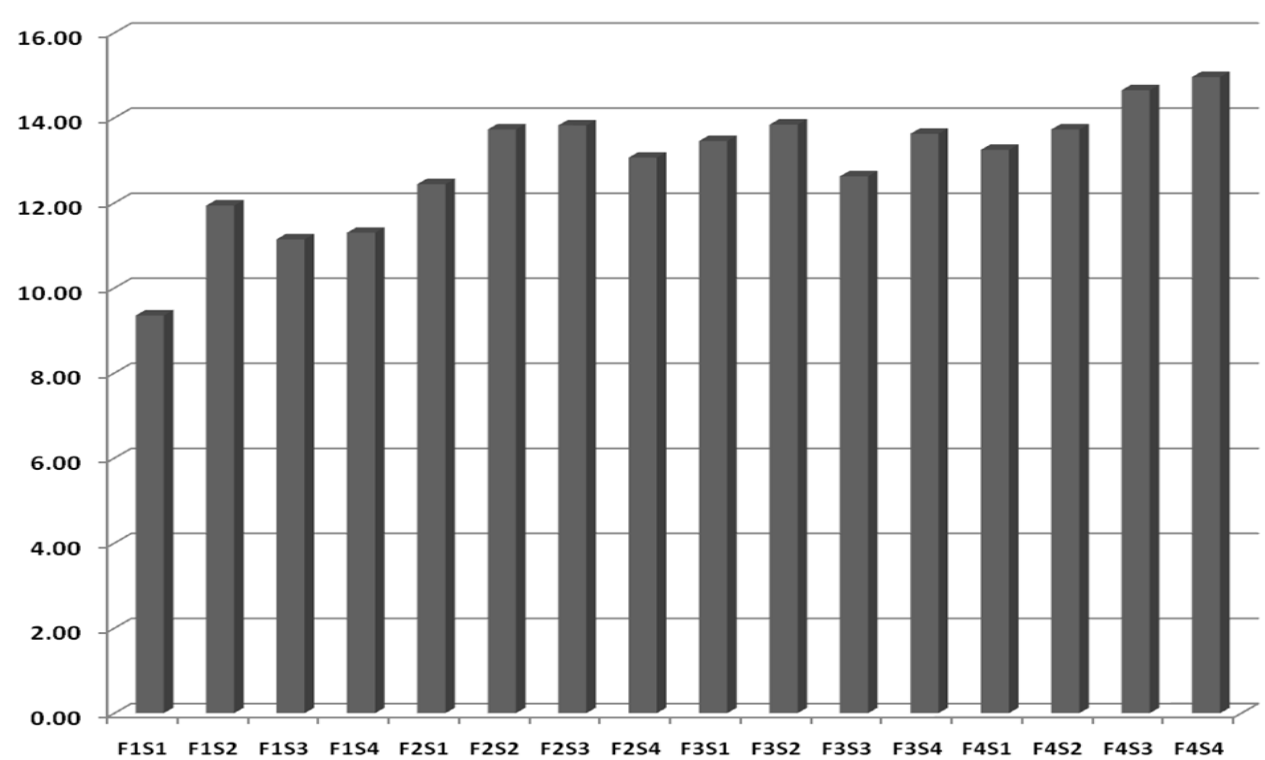

Fig. 1. Yield of Brinjal observed under different nutrient management protocols. $F_{1}-125: 100: 50(R D F), F_{2}-75 \% R D F+$ Azotobacter, $F_{3^{-}} 75 \% R D F+P S B, F_{4^{-}} 75 \% R D F+$ Azotobacter + PSB; $S_{1^{-}}$Control, $S_{2^{-}} \mathrm{ZnSO}_{4} @ 25 \mathrm{~kg} / \mathrm{ha}, \mathrm{S}_{3^{-}} \mathrm{FeSO}_{4} @ 50 \mathrm{~kg} / \mathrm{ha}, \mathrm{S}_{4^{-}} \mathrm{Bo}$ rax@10kg/ha. 
Table 4. Organic matter and nutrient status of the post-harvest soil of brinjal under varying protocols of integrated nutrient management

\begin{tabular}{|c|c|c|c|c|c|c|c|c|}
\hline Treatment & $\begin{array}{l}\text { Organic } \\
\text { C }(\mathrm{g} / \mathrm{kg}) \\
\end{array}$ & $\begin{array}{l}\text { Total N } \\
(\mathrm{g} / \mathrm{kg})\end{array}$ & $\begin{array}{l}\text { Available } \\
\text { N (kg/ha) }\end{array}$ & $\begin{array}{l}\text { Available } \\
\text { P (kg/ha) }\end{array}$ & $\begin{array}{l}\text { Available } \\
\text { K (kg/ha) }\end{array}$ & $\begin{array}{l}\text { Available } \\
\text { Fe (kg/ha) }\end{array}$ & $\begin{array}{l}\text { Available } \\
\text { Zn (kg/ha) }\end{array}$ & $\begin{array}{l}\text { Available } \\
\text { B (kg/ha) }\end{array}$ \\
\hline \multicolumn{9}{|c|}{ Fertilizers and Bio-fertilizers } \\
\hline$\overline{F_{1}}$ & $4.95^{\mathrm{c}}$ & $0.66^{\mathrm{b}}$ & $180.75^{\mathrm{b}}$ & $60.08^{\mathrm{c}}$ & $147.77^{\mathrm{a}}$ & $22.17^{\mathrm{d}}$ & $4.51^{\mathrm{d}}$ & $0.50^{\mathrm{c}}$ \\
\hline $\mathrm{F}_{2}$ & $7.05^{\mathrm{b}}$ & $0.89^{\mathrm{a}}$ & $197.82^{\mathrm{a}}$ & $65.09^{\mathrm{b}}$ & $157.18^{\mathrm{a}}$ & $24.64^{\mathrm{b}}$ & $5.58^{\mathrm{c}}$ & $0.60^{\mathrm{b}}$ \\
\hline $\mathrm{F}_{3}$ & $6.74^{\mathrm{b}}$ & $0.75^{\mathrm{b}}$ & $192.14^{\mathrm{ab}}$ & $73.17^{\mathrm{a}}$ & $152.60^{\mathrm{a}}$ & $25.58^{\mathrm{a}}$ & $6.50^{\mathrm{b}}$ & $0.61^{\mathrm{b}}$ \\
\hline $\mathrm{F}_{4}$ & $7.73^{\mathrm{a}}$ & $0.95^{\mathrm{a}}$ & $199.79^{\mathrm{a}}$ & $68.60^{\mathrm{b}}$ & $164.48^{\mathrm{a}}$ & $23.94^{\mathrm{c}}$ & $6.77^{\mathrm{a}}$ & $0.70^{\mathrm{a}}$ \\
\hline \multicolumn{9}{|c|}{ Micronutrients } \\
\hline $\mathrm{S}_{1}$ & $6.34^{\mathrm{a}}$ & $0.71^{\mathrm{b}}$ & $186.93^{\mathrm{a}}$ & $63.22^{\mathrm{b}}$ & $151.51^{\mathrm{a}}$ & $23.27^{\mathrm{d}}$ & $4.77^{\mathrm{c}}$ & $0.52 \mathrm{c}$ \\
\hline $\mathrm{S}_{2}$ & $6.60^{\mathrm{a}}$ & $0.81^{\mathrm{a}}$ & $192.48^{\mathrm{a}}$ & $68.27^{\mathrm{a}}$ & $148.88^{\mathrm{a}}$ & $23.50^{\mathrm{c}}$ & $6.34^{\mathrm{a}}$ & $0.60^{\mathrm{b}}$ \\
\hline $\mathrm{S}_{3}$ & $6.69^{\mathrm{a}}$ & $0.82^{\mathrm{a}}$ & $195.46^{\mathrm{a}}$ & $66.63^{\mathrm{ab}}$ & $159.57^{\mathrm{a}}$ & $25.44^{\mathrm{a}}$ & $6.18^{\mathrm{ab}}$ & $0.60^{\mathrm{b}}$ \\
\hline $\mathrm{S}_{4}$ & $6.84^{\mathrm{a}}$ & $0.90^{\mathrm{a}}$ & $195.63^{\mathrm{a}}$ & $68.81^{\mathrm{a}}$ & $162.07^{\mathrm{a}}$ & $24.13^{\mathrm{b}}$ & $6.07^{\mathrm{b}}$ & $0.69^{\mathrm{a}}$ \\
\hline \multicolumn{9}{|c|}{ Interaction (F X S) } \\
\hline$\overline{F_{1} S_{1}}$ & $4.46^{\mathrm{d}}$ & $0.49^{\mathrm{e}}$ & $173.16^{\mathrm{b}}$ & $53.79^{\mathrm{c}}$ & $129.14^{\mathrm{a}}$ & $20.55^{\mathrm{k}}$ & $3.03^{\mathrm{g}}$ & $0.40^{\mathrm{f}}$ \\
\hline $\mathrm{F}_{1} \mathrm{~S}_{2}$ & $4.95^{\mathrm{d}}$ & $0.54^{\mathrm{de}}$ & $181.66^{\mathrm{ab}}$ & $61.21^{\mathrm{bc}}$ & $148.10^{\mathrm{a}}$ & $22.07^{\mathrm{i}}$ & $4.32^{\mathrm{e}}$ & $0.52^{\mathrm{de}}$ \\
\hline $\mathrm{F}_{1} \mathrm{~S}_{3}$ & $5.13^{\mathrm{cd}}$ & $0.73^{\text {bcde }}$ & $187.94^{\mathrm{ab}}$ & $62.85^{\mathrm{bc}}$ & $160.16^{\mathrm{a}}$ & $24.10^{\mathrm{g}}$ & $5.40^{\mathrm{d}}$ & $0.48^{\mathrm{ef}}$ \\
\hline $\mathrm{F}_{1} \mathrm{~S}_{4}$ & $5.25^{\mathrm{bcd}}$ & $0.88^{\mathrm{abc}}$ & $180.24^{\mathrm{b}}$ & $62.45^{\mathrm{bc}}$ & $153.67^{\mathrm{a}}$ & $21.95^{\mathrm{i}}$ & $5.30^{\mathrm{d}}$ & $0.60^{\text {cd }}$ \\
\hline $\mathrm{F}_{2} \mathrm{~S}_{1}$ & $6.76^{\mathrm{ab}}$ & $0.82^{\mathrm{abcd}}$ & $198.66^{\mathrm{ab}}$ & $65.62^{\mathrm{abc}}$ & $168.23^{\mathrm{a}}$ & $24.36^{\mathrm{f}}$ & $3.72^{\mathrm{f}}$ & $0.53^{\mathrm{de}}$ \\
\hline $\mathrm{F}_{2} \mathrm{~S}_{2}$ & $7.05^{\mathrm{a}}$ & $0.87^{\mathrm{abc}}$ & $190.70^{\mathrm{ab}}$ & $67.49^{\mathrm{ab}}$ & $149.00^{\mathrm{a}}$ & $24.63^{\mathrm{e}}$ & $6.83^{\mathrm{b}}$ & $0.57^{\mathrm{de}}$ \\
\hline $\mathrm{F}_{2} \mathrm{~S}_{3}$ & $7.13^{\mathrm{a}}$ & $0.91^{\mathrm{abc}}$ & $201.23^{\mathrm{ab}}$ & $61.76^{\mathrm{bc}}$ & $150.08^{\mathrm{a}}$ & $25.24^{\mathrm{c}}$ & $5.45^{\mathrm{d}}$ & $0.62^{\mathrm{bcd}}$ \\
\hline $\mathrm{F}_{2} \mathrm{~S}_{4}$ & $7.24^{\mathrm{a}}$ & $0.94^{\mathrm{abc}}$ & $200.71^{\mathrm{ab}}$ & $65.50^{\mathrm{abc}}$ & $161.41^{\mathrm{a}}$ & $24.34^{\mathrm{f}}$ & $6.30^{\mathrm{c}}$ & $0.68^{\mathrm{abc}}$ \\
\hline $\mathrm{F}_{3} \mathrm{~S}_{1}$ & $6.63^{\mathrm{abc}}$ & $0.68^{\text {cde }}$ & $188.00^{\mathrm{ab}}$ & $71.44^{\mathrm{ab}}$ & $150.66^{\mathrm{a}}$ & $24.84^{\mathrm{d}}$ & $5.74^{\mathrm{d}}$ & $0.55^{\mathrm{de}}$ \\
\hline $\mathrm{F}_{3} \mathrm{~S}_{2}$ & $6.70^{\mathrm{ab}}$ & $0.84^{\mathrm{abc}}$ & $198.15^{\mathrm{ab}}$ & $72.68^{\mathrm{ab}}$ & $149.88^{\mathrm{a}}$ & $26.14^{\mathrm{b}}$ & $6.60^{\mathrm{bc}}$ & $0.57^{\mathrm{de}}$ \\
\hline $\mathrm{F}_{3} \mathrm{~S}_{3}$ & $6.83^{\mathrm{a}}$ & $0.75^{\text {bcde }}$ & $193.53^{\mathrm{ab}}$ & $72.35^{\mathrm{ab}}$ & $154.26^{\mathrm{a}}$ & $26.08^{\mathrm{b}}$ & $7.41^{\mathrm{a}}$ & $0.60^{\mathrm{cd}}$ \\
\hline $\mathrm{F}_{3} \mathrm{~S}_{4}$ & $6.81^{\mathrm{a}}$ & $0.73^{\text {bcde }}$ & $188.91^{\mathrm{ab}}$ & $76.20^{\mathrm{a}}$ & $155.61^{\mathrm{a}}$ & $25.28^{\mathrm{c}}$ & $6.26^{\mathrm{c}}$ & $0.71^{\mathrm{ab}}$ \\
\hline $\mathrm{F}_{4} \mathrm{~S}_{1}$ & $7.51^{\mathrm{a}}$ & $0.83^{\mathrm{abc}}$ & $187.91^{\mathrm{ab}}$ & $62.06^{\mathrm{bc}}$ & $158.00^{\mathrm{a}}$ & $23.31^{\mathrm{h}}$ & $6.59^{\mathrm{bc}}$ & $0.60^{\mathrm{cd}}$ \\
\hline $\mathrm{F}_{4} \mathrm{~S}_{2}$ & $7.68^{\mathrm{a}}$ & $1.01^{\mathrm{ab}}$ & $199.43^{\mathrm{ab}}$ & $71.69^{\mathrm{ab}}$ & $148.54^{\mathrm{a}}$ & $21.15^{\mathrm{j}}$ & $7.62^{\mathrm{a}}$ & $0.74^{\mathrm{a}}$ \\
\hline $\mathrm{F}_{4} \mathrm{~S}_{3}$ & $7.67^{\mathrm{a}}$ & $0.90^{\mathrm{abc}}$ & $199.15^{\mathrm{ab}}$ & $69.55^{\mathrm{ab}}$ & $173.80^{\mathrm{a}}$ & $26.34^{\mathrm{a}}$ & $6.45^{\mathrm{bc}}$ & $0.69^{\mathrm{abc}}$ \\
\hline $\mathrm{F}_{4} \mathrm{~S}_{4}$ & $8.04^{\mathrm{a}}$ & $1.05^{\mathrm{a}}$ & $212.67^{\mathrm{a}}$ & $71.08^{\mathrm{ab}}$ & $177.59^{\mathrm{a}}$ & $24.94^{\mathrm{d}}$ & $6.43^{\mathrm{bc}}$ & $0.78^{\mathrm{a}}$ \\
\hline
\end{tabular}

$\mathrm{F}_{1}-125: 100: 50(\mathrm{RDF}), \mathrm{F}_{2}-75 \% \mathrm{RDF}+$ Azotobacter, $\mathrm{F}_{3}-75 \% \mathrm{RDF}+\mathrm{PSB}, \mathrm{F}_{4}-75 \% \mathrm{RDF}+$ Azotobacter $+\mathrm{PSB} ; \mathrm{S}_{1}-\mathrm{Control}, \mathrm{S}_{2}-$ $\mathrm{ZnSO}_{4} @ 25 \mathrm{~kg} / \mathrm{ha}, \mathrm{S}_{3}-\mathrm{FeSO}_{4} @ 50$ kg/ha, $\mathrm{S}_{4}-$ Borax @10 kg/ha; a, b different superscriptions against means denote significant differences (otherwise statistically at par) at $\mathrm{P}<0.05$ by Tukey's Honest significant Difference Test

173.16 (control) to 212.67 (75\% RDF + Azospirillum + PSB + Borax@10 kg ha $\left.{ }^{-1}\right)$. The significant increase in total $\mathrm{N}$ of post harvest soils receiving NPK and Azospirillum remained quite imperative due to further $\mathrm{N}$ addition through biological fixation (Table 4).

Available $\mathrm{P}$ content $\left(\mathrm{kg} \mathrm{ha}^{-1}\right)$ of post harvest soil of brinjal has been observed to vary within a range of 53.79 (control) to 76.20 (75\% RDF + Azospirillum + PSB + Borax@10 kg ha-1) (Table 4).The maximum recoveries of available $\mathrm{P}$ has been found to be associated with brinjal seedling inoculation through PSB which might be contributed to available $\mathrm{P}$ pool of the soil through enhanced microbe-mediated dissolution of not -so-available soil $\mathrm{P}$ fractions (non-exchangeable, adsorbed etc.). It has been earlier noted by several workers that it was also observed that the biological fertilizer application releases certain chemical compounds which in turn increases the phosphorus solubility (Yousefi et al., 2011)

Available potassium $\left(\mathrm{kg} \mathrm{ha}^{-1}\right)$ of the post harvest soil of brinjal has been observed to vary within a range of
129.04 (control) to 177.59 (75\% RDF + Azospirillum + PSB + Borax@10 kg ha ${ }^{-1}$ ) (Table 4). The availabilities of $\mathrm{P}$ and $\mathrm{K}$ were found higher with bacterial fertilizers (N-fixers), since the build-up of available $\mathrm{P}$ and $\mathrm{K}$ in the soil could be due to the organic acids which were released during microbial decomposition of native soil organic fractions increasing the available $\mathrm{P}$ and $\mathrm{K}$ in soil (Choudhury et al., 2005). Available iron $\left(\mathrm{kg} \mathrm{ha}^{-1}\right)$ in the post harvest soil of brinjal ranged from 20.55 (control) to 26.14 (75\% RDF + Azospirillum + PSB + $\left.\mathrm{FeSO}_{4} @ 50 \mathrm{~kg} \mathrm{ha}^{-1}\right)$. The maximum recoveries of available $\mathrm{Fe}$ has been found to be associated with application of iron fertilizers $\left(\mathrm{FeSO}_{4} @ 50 \mathrm{~kg} \mathrm{ha}^{-1}\right)$ along with NPK and bacterial fertilizers. Such increases [\% over control (RDF)] have also been supported by Fe-fertilizer application over NPK + Bacterial fertilizers (Table 4). Available zinc content $\left(\mathrm{kg} \mathrm{ha}^{-1}\right)$ of the post harvest soil of brinjal ranged from 3.03 (control) to 7.62 (75\% RDF + Azospirillum + PSB $+\mathrm{ZnSO}_{4} @ 25 \mathrm{~kg} \mathrm{ha}^{-1}$ ). The maximum recoveries of available $\mathrm{Zn}$ has been found to be associated with 
application of iron fertilizers $\left(\mathrm{ZnSO}_{4} @ 25 \mathrm{~kg} \mathrm{ha}^{-1}\right)$ along with NPK and bacterial fertilizers. Such increases $[\%$ over control (RDF)] have also been supported by Zn-fertilizer application over NPK + bacterial fertilizers (Table 4). Available boron content $\left(\mathrm{kg} \mathrm{ha}^{-1}\right)$ of the post harvest soil of brinjal ranged from 0.40 (control) to 0.78 (75\% RDF + Azospirillum + PSB + Borax@10 kg ha ${ }^{-1}$ ). The maximum recoveries of available $\mathrm{B}$ has been found to be associated with application of boron fertilizers (Borax@10 kg/ha) along with NPK and bacterial fertilizers. Such increases $[\%$ over control (RDF)] have also been supported by borax application over NPK + Bacterial fertilizers (Table 4).

The yield of brinjal cultivated with interventions of the selected nutrient management practices are shown in Fig. 1. Brinjal yields supported by different INM protocols ranged from 9.35 (control) to $14.96 \mathrm{t} \mathrm{ha}^{-1}$ (75\% recommended dose of fertilizer NPK (RDF i.e. $\mathrm{N}: \mathrm{P}: \mathrm{K}::$ 125:100:50) + Azospirillum + PSB + Boron @ $\left.10 \mathrm{Kg} \mathrm{ha}^{-1}\right)$ (Fig 1). Results revealed that integrated management of nutrients through supplementation of chemical fertilizers with bacterial fertilizers along with micronutrient fertilizers brought significant enhancement in Brinjal fruit yields over the control counterparts (RDF only). Managing the nutrient requirement of Brinjal through a combined application of $75 \% \mathrm{RDF}+$ Azospirilum + PSB + Boron@10 $\mathrm{kg} \mathrm{ha}^{-1}$ recorded the highest yield followed by the treatment combination of $75 \% \mathrm{RDF}+$ Azospirillum + PSB + $\mathrm{FeSO}_{4} @ 50 \mathrm{Kg} \mathrm{ha}$ 1. Maximum increments in Brinjal yields obtained remained to the tune of $60 \%$ over control. Such results are in good agreement with Yadav et al. (2006).

\section{Conclusion}

It is concluded that the treatment consisting of $75 \%$ RDF (RDF i.e. N:P:K:: 125:100:50) + Azospirillum + PSB + Borax@10 kg ha ${ }^{-1}$ recorded the highest brinjal yield, oxidisable organic carbon $\left(8.049 \mathrm{~g} \mathrm{~kg}^{-1}\right)$, total nitrogen $\left(1.05 \mathrm{~g} \mathrm{~kg}^{-1}\right)$, available nitrogen $\left(212.67 \mathrm{~g} \mathrm{~kg}^{-1}\right)$, available phosphorus $\left(76.20 \mathrm{~g} \mathrm{~kg}^{-1}\right)$ and available potassium $\left(177.59 \mathrm{~g} \mathrm{~kg}^{-1}\right)$ in the post harvest soils of brinjal. Integrated management of nutrients through supplementation of chemical fertilizers by bacterial fertilizers along with micronutrients inputs was found to significantly increase available micro-nutrient status of the post harvest soil.

\section{REFERENCES}

AOAC, (1995). Official methods of analysis. 16th edn Association of Official Analytical Chemists, Washington, DC.

Bashan, Y., Holguinm, G. and de-Bashanl, L.E. (2004). Azospiriilum- plant relationships: physiological, molecular, agricultural and environmental advances (1997-2003). Canadian Journal of Microbiology. 50: 521-577.

Bashyal, L.N. (2011). Response of cauliflower to nitrogen fixing biofertilizer and graded levels of nitrogen.
Journal of Agriculture and Environment, 12: 41-50.

Berger, K.C. and Troug, K. (1939). Boron determined by using the quinalizarin reaction, Ind. Eng. Chem II : 540 545 .

Bouyoucos, G.J. (1962). Hydrometer method improved for making particle size analysis of soils. Agronomy Journal, 54, 464-465.

Brown, A.J. and Warncke, D. (1988). In W.C. Dahnke (ed.) Recommended chemical soil test procedures for the North Carolina Region. North Dakota Agricultural Experimental Station Bull, 499, 15-16.

Choudhury, M.R., Talukdar, N.C. and Saikia, A. (2005). Effect of integrated nutrient management on growth and productivity of brinjal. Research on Crops, 6(3): 551554.

Daunay, M.C., Jullian, E. and Dauphin, F. (2001). Management of eggplant and pepper genetic resources in Europe: networks are emerging. Proceedings of 11th EUCARPIA meeting on genetics and breeding of Capsicum and eggplant, 9-13,April 2001,Antalya, Turkey, Adana, Turkey. pp. 1-5.

Gaines, T.P. and G.A. Mitchell. 1979. Boron determination in plant tissue by the azomethine-H method. Communication in Soil Science and Plant Analysis, 10: 1099. 1108 .

Hedge, D.M. (1997). Nutrient requirement of Solanaceous vegetable crops. Ext. Bull. 441. Food fertilizer technology center. Asian and Pacific Council, Berkeley, CA.

Jackson, M.L. (1967). Soil Chemical Analysis, Prentice Hall of India Pvt. Ltd., New Delhi, India.

Jackson, M.L. (1973). Soil chemical analysis. Prentice Hall of India Pvt. Ltd., New Delhi, pp. 48-341.

Kadlag, A.D., Jadhav, A.B. and Bharti, R. (2007) Yield and quality of tomato fruit as influenced by biofertilizers. An Asian Journal of Soil Science, 2(2): 95-99

Karuppaiah, P. (2005). Foliar application of micronutrients on growth, flowering and yield characters of brinjal cv. Annamalai. Plant Archives, 5(2): 605-608.

Khan, M.S., Shil, N.C., and Noor, S. (2008). Integrated Nutrient Management for Sustainable Yield of Major Vegetable Crops in Bangladesh. Bangladesh Journal of Agriculture and Environment, 4: 81-94.

Kiran, J., Vyakarana, B.S., Raikar, S.D., Ravikumar, G.H. and Deshpande, V.K. (2010). Seed yield and quality of brinjal as influenced by crop nutrition. Indian Journal of Agricultural Research, 44(1): 1-7.

Kumar Mohan, A.B., Narase, N.C., Raviraja, G., Shetty, G. and Kartik, N.M. (2011) Effect of organic manures and inorganic fertilizers on available NPK, microbial density of the soil and nutrient uptake of Brinjal. Research Journal of Agriculture Science, 2(2): 304-307.

Ladha, P., Jeyaraman, S and Prabakaran, R. (2014). Effect of Microbial and Chemical Fertilizer on Egg Plant (Solanum melongena LINN.) C.Var CO-2. International Journal of Pure \& Applied Bioscience, 2(4): 119-124.

Lindsay, W.L., Norvell, W.A. (1978). Development of DTPA soil test for $\mathrm{Zn}, \mathrm{Fe}, \mathrm{Mn}$ and $\mathrm{Cu}$. Soil Science Society of America Journal, 42: 421-428.

Olsen, S.R., Cole, C.V., Watanale, F.S., Dean, L.A. (1954). Estimation of available phosphorus in phosphorus in soils by extraction with sodium bicarbonate. Circular 393, United States Department of Agriculture, Washington DC.

Patil, G.D. and Patil, B.R. (2000). Mineral composition of new brinjal cultivars. Journal of Maharastra Agricultural 
Universities, 25: 210-211.

Rajan, S. and Markose, B.L. (2002). Propagation of Horticultural Crops. Horticultural Science Series, 6: 94.

Sadasivam, S. and Manickam, A. (1996). Biochemical methods. Book New Age International (P) Limited, Publishers, second edition, May. pp. 34-37.

Subbiah, B. and Asija, G.L. (1956). A rapid procedure for the estimation of available $\mathrm{N}$ in soils. Current Science, 25: 259-260.

Saravaiya, S.N. and Patel, M.B. (2005). DNM (Diamond black moth): the most notorious pest of cauliflower and its management strategies. Agrobios Newsletter, 3: 2324.

Srijaya, T. and Sitaramayya, J. (2006). Effect of nutrient management on yield and nutrient content of brinjal grown in an Alfisol, Ranga Reddy District, A.P. Journal of Soils and Crops, 16(2): 264-269.

Selvi, D., Thiageshwari, S., Santhy, P. and Kannan, B.R. (2004). Fruit yield and nutrient uptake by brinjal due to integrated nutrient management in an Inceptisol. Journal of Maharastra Agricultural Universities, 29(2): 220-223.

Sharma, J.P. (2011). Organic Vegetables: production and protection technology In: quality seed production of vegetable crops. Vol. I (Ed. J. P. Sharma). Kalyani Publishers New Delhi, pp. 123-143.

Sharma J.P., Rattan P and Kumar, S. (2012). Response of vegetable crops to use of integrated nutrient management practices. SABB Journal of Food and Agriculture Science, 2(1): 15-19.

Singh, S., Zacharias. M., Kalpana, S. and Mishra. M. (2012). Heavy metals accumulation and distribution pattern in different vegetable crops. Journal of Enviromental Chemistry and Ecotoxicology, 4(4): 75-81.

Singh, T.R., Singh, S., Singh, S.K. and Singh, M.P. (2004). Influence of integrated nutrient management on properties of a Mollisol under okra-pea-tomato cropping sequence. Agropedology, 14(2): 92-95.

Yadav, M., Singh, D.B., Chaudhary, R. and Reshi, T.A. (2006). Effect of boron on yield of tomato (Lycopersicon esculentum Mill) cv. DVRT-1. Plant Archives, 6(1): 383-384.

Yousefi, A.A., Khavazi, K., Moezi, A.A., Rejali, F. and Nadian, H.A. (2011). Phosphate solubilizing bacteria and Arbuscular mycorrhizal fungi impacts on inorganic phosphorus fractions and wheat growth. World Applied Sciences Journal, 15(9): 1310-1318. 